\title{
Impact of variation in patient response on model-based control of glycaemia in critically ill patients
}

\author{
Aaron J. Le Compte*, Christopher G. Pretty*, Jessica Lin**, Geoffrey M. Shaw***, \\ Adrienne Lynn****, J. Geoffrey Chase* \\ *Department of Mechanical Engineering, University of Canterbury, Christchurch, New Zealand (email: \\ aaron.lecompte@canterbury.ac.nz,christopher.pretty@pg.canterbury.ac.nz,geoff.chase@canterbury.ac.nz) \\ ** Department of Medicine, University of Otago, Christchurch, New Zealand (email: jessica.lin@otago.ac.nz) \\ ***Department of Intensive Care, Christchurch Hospital, Christchurch, New Zealand (email: geoff.shaw@cdhb.govt.nz) \\ ****Neonatal Department, Christchurch Women's Hospital, Christchurch, New Zealand (email: adrienne.lynn@cdhb.govt.nz)
}

\begin{abstract}
Critically ill patients commonly experience stress-induced hyperglycaemia, and several studies have shown tight glycaemic control (TGC) can reduce patient mortality. However, tight control is often difficult to achieve due to conflicting drug therapies and evolving patient condition. Thus, a number of studies have failed to achieve TGC possibly due to use of fixed insulin dosing protocols over adaptive patient-specific methods. Model-based targeted glucose control can adapt insulin and dextrose interventions to match identified patient sensitivity. This study explores the impact on control of assuming patient response to insulin is constant versus time-varying. Simulated trials of glucose control were performed on adult and neonatal virtual patient cohorts. Results indicate assumptions of constant insulin sensitivity can lead to significantly increased rates of hypoglycaemia, a commonly cited issue preventing increased adoption of tight glycaemic control in critical care. It is clear that adaptive, patientspecific, approaches are better able to manage inter- and intra- patient variability than typical, fixed protocols.
\end{abstract}

Keywords: Critical care, glycaemic control, simulation, modelling, insulin sensitivity

\section{INTRODUCTION}

Critically ill patients, both adult and infant, often experience hyperglycaemia and high levels of resistance to insulin (McCowen et al., 2001). Hyperglycaemia worsens outcomes, increasing the risk of severe infection (Bistrian, 2001), myocardial infarction (Capes et al., 2000) and critical illness such as polyneuropathy and multiple organ failure (Van den Berghe et al., 2001). The occurrence of hyperglycaemia is associated with increased morbidity and mortality in adults. Glycaemia variability, and thus poor control, is also independently associated with increased mortality (Egi et al., 2006).

An increasing body of recent literature links hyperglycaemia in preterm neonates to worsened outcomes in a parallel of the adult case. Studies have demonstrated an increased risk of further complications such as sepsis, increased ventilator dependence, retinopathy of prematurity, hospital length of stay and mortality associated with high levels of blood glucose (Alaedeen et al., 2006, Hays et al., 2006, Ertl et al., 2006).

Hyperglycaemia as a response to the stress of critical illness is a common origin of this altered metabolic state in both adults and neonates. The counter-regulatory response to stress increases the level of circulating catecholamines, resulting in increased endogenous glucose production and reduced sensitivity to insulin. Hyperglycaemia in the neonate is unique in that in addition to manifestation as a response to stress, several patho-physiologies are directly related to the immaturity of the glucose regulatory system, including impaired beta-cell secretion of insulin (Mitanchez-Mokhtari et al., 2004), limited number of insulin-dependent tissues (Raney et al., 2008) and hepatic unresponsiveness to glucose infusions (Cowett et al., 1983).

Tight glycaemic control has been shown to reduce mortality by $18-45 \%$ in adult patients (Chase et al., 2008, Van den Berghe et al., 2001). There is also evidence of significant reductions in the need for dialysis, bacteraemia testing and blood transfusions with TGC using intensive insulin therapy (Van den Berghe et al., 2001). All of these results point towards the conclusion that the control of blood glucose levels in adult critical care has a significant clinical impact.

Although it is now becoming an unacceptable practice to allow hyperglycaemia and its associated effects (Preiser and Devos, 2007), moderately elevated blood glucose levels are tolerated or recommended (Schultz et al., 2008) because of the fear of hypoglycaemia and higher nursing effort frequently associated with TGC (Preiser and Devos, 2007). Model-based control may offer advantages in glucose regulation (Chase et al., 2006).

In general, any glycemic control protocol must reduce elevated blood glucose levels with minimal hypoglycaemia, while accounting for inter-patient variability, conflicting drug 


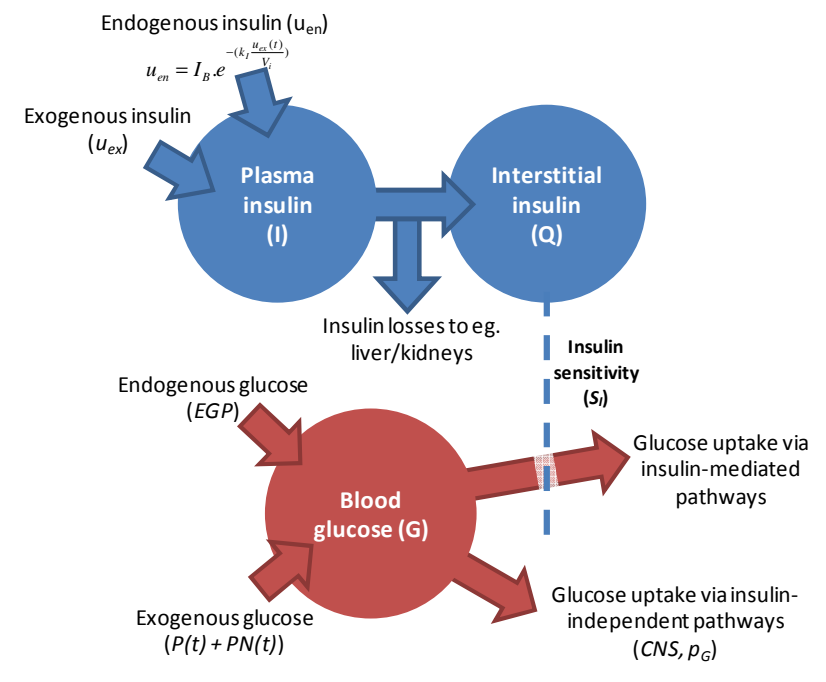

Fig 1. Major components of the glucose-insulin model.

therapies and dynamically evolving physiological condition. Thus, it must titrate glucose control interventions based on some estimate of patient metabolic state - either identified from data in the case of adaptive model-based control, or assumed constant in some form as in the case of fixed insulin dosing protocols.

In this study, the effects of intra- and inter- patient variability in sensitivity to insulin is explored in the context of simulations of glucose control using a clinically validated glucose-insulin system model. Adaptive, model-based control is modified in simulation to test the relative importance of tracking metabolic state between patients and over time by instructing the controller to assume the patient response to insulin is constant.

Cohorts of virtual patients, fitted from clinical retrospective data, are used to determine the impact of not adequately addressing inter- or intra-patient variability on glycaemic control. Clinically validated virtual trial simulations are performed on both adult and neonatal patients to highlight the potential for model-based control to better adapt to significantly different clinical situations.

Many insulin therapy regimes employ fixed dosing protocols, or dosing schemes adjusted by patient weight or other factors (Beardsall et al., 2007), and thus ignore inter- and/or intrapatient variability in metabolic response. Thus, exploring the relative importance of model-based control to account for inter- vs. intra- patient variabilities and the differences in variability between adults and neonates can indicate the mechanisms by which model-based control can provide more robust and safer control of glucose levels.

\section{METHODS}

\subsection{Models}

Blood glucose system models clinically validated in both adults and neonates are used in this study (Chase et al., 2007). The overall form of the models is presented in Equations (1)-(3). Major components of the model are displayed in Fig 1.

$$
\begin{aligned}
& \dot{G}=-p_{G} \cdot G-S_{I} \cdot G \cdot \frac{Q}{1+\alpha_{G} Q} \\
& +\frac{P(t)+\left(P_{E N D} * m_{\text {body }}\right)-\left(C N S * m_{\text {brain }}\right)}{\left(V_{G, \text { frac }}(t) * m_{\text {body }}\right)}
\end{aligned}
$$

$\dot{Q}=-k Q+k I$

$\dot{I}=-\frac{n I}{1+\alpha_{I} I}+\frac{u_{e x}(t)}{\left(V_{I, \text { frac }} * m_{\text {body }}\right)}+I_{B} \cdot e^{-\left(k_{I} \frac{u_{e x}(t)}{V_{i}}\right)}$

Where $G(t)[\mathrm{mmol} / \mathrm{L}]$ is plasma glucose $I(t)[\mathrm{mU} / \mathrm{L}]$ is plasma insulin, $u_{e x}(t)[\mathrm{mU} / \mathrm{min}]$ is exogenous insulin input, basal endogenous insulin secretion is $I_{B}[\mathrm{mU} / \mathrm{L} / \mathrm{min}]$, with $k_{I}$ representing suppression of basal insulin secretion by exogenous insulin. Interstitial insulin is $Q(t)$ [mU/L], with $k$ [1/min] accounting for losses and transport. Body weight and brain weight are denoted by $m_{\text {body }}[\mathrm{kg}]$ and $m_{\text {brain }}[\mathrm{kg}]$. Patient endogenous glucose clearance and insulin sensitivity are $p_{G}$ [1/min] and $S_{I}[\mathrm{~L} /(\mathrm{mU} \cdot \mathrm{min})]$. The parameter $V_{I, \text { frac }}[\mathrm{L} / \mathrm{kg}]$ is the insulin distribution volume per $\mathrm{kg}$ body weight and $n$ [1/min] is the transport rate of insulin from plasma. Total plasma glucose input is $P(t) \quad[\mathrm{mmol} / \mathrm{min}]$, endogenous glucose production is $P_{E N D}[\mathrm{mmol} / \mathrm{kg} / \mathrm{min}]$ and $V_{G, f r a c}[\mathrm{~L} / \mathrm{kg}]$ represents the glucose distribution volume per $\mathrm{kg}$ body weight. CNS [mmol $/ \mathrm{kg} / \mathrm{min}]$ captures non-insulin mediated glucose uptake by the central nervous system. MichaelisMenten functions model saturation, with $\alpha_{I}[\mathrm{~L} / \mathrm{mU}]$ for the saturation of plasma insulin disappearance, and $\alpha_{G}[\mathrm{~L} / \mathrm{mU}]$ for insulin-dependent glucose clearance saturation.

The models are similar in structure, with modifications generally in parameter values to account for differences in adult and neonatal physiology, and to account for the number of kinetic studies available in the neonatal literature. Further details on model development (Hann et al., 2005), simulation (Lonergan et al., 2006) and clinical usage (Wong et al., 2006) are available elsewhere.

\subsection{Patient cohorts}

For this study, two cohorts are analysed:

- Adult ICU (ICU): N = 393 patients from SPRINT with over 40,000 hours of data (Chase et al., 2008)

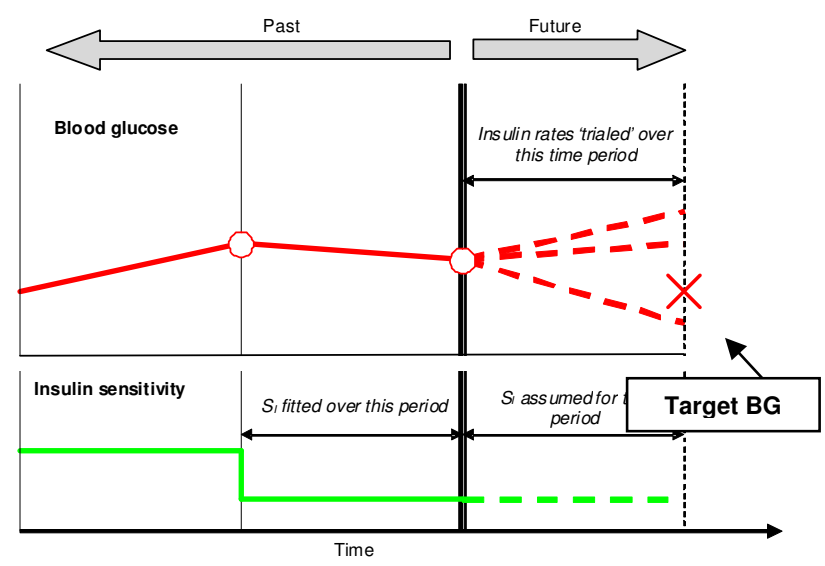

Fig 2. Adaptive control methodology. 

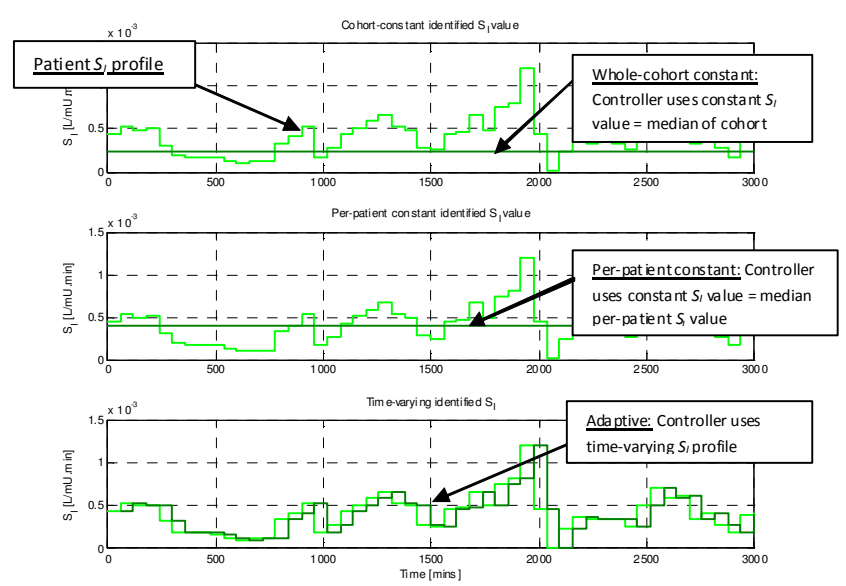

Fig 3. Comparison of whole-cohort constant $S_{I}$, per-patient constant $S_{I}$ and adaptive control methods.

- $\quad$ Neonatal ICU (NICU): $\mathrm{N}=25$ patients and over 3,500 hours of data (Le Compte et al., 2009)

For each cohort, insulin sensitivity, $S_{I}$, is identified hourly from the clinical data. A virtual patient profile is the record of fitted insulin sensitivity. The fit and prediction ability of the models is similar over both cohorts, with a median blood glucose fit error of $1.2 \%$ (IQR: $0.5-2.0 \%$ ) and $2.4 \%$ (IQR: $0.9-4.8 \%$ ) for the adult and neonatal cohorts respectively. Blood glucose prediction errors in response to insulin and dextrose interventions for the adult and neonatal models are also similar with $5-10 \%$ BG errors for 1 and 2 hour forward windows respectively.

\subsection{Simulated trials and control methods}

Adaptive control was performed in simulation across both cohorts using the control methodology, as described in Fig 2, to mimic typical clinical usage. For adaptive control the insulin sensitivity parameter $\left(S_{I}\right)$ is fitted over the patient data up to the current BG measurement at each BG measurement and control intervention cycle. This value of insulin sensitivity is held constant over the prediction interval and Equations (1) - (3) are solved for 1 to 2 hours into the future. The glucose response to a range of insulin values is computed to determine which insulin rate would achieve control closest to the target BG concentration. Blood glucose targets were a reduction of $15 \%$ per hour, to a minimum target of 5.0 $\mathrm{mmol} / \mathrm{L}$ for adults, and $6.0 \mathrm{mmol} / \mathrm{L}$ for neonates.

To explore the impact of variation in insulin sensitivity on model-based control, the real-time fitting of insulin sensitivity was replaced by two alternatives and compared to the adaptive control method, summarised in Fig 3:

- Per-patient constant insulin sensitivity: The median fitted value of insulin sensitivity for each patient is used as the value of $S_{I}$ employed by the controller in choosing insulin rates. This control method accounts for inter- patient, but not intra- patient, variability.

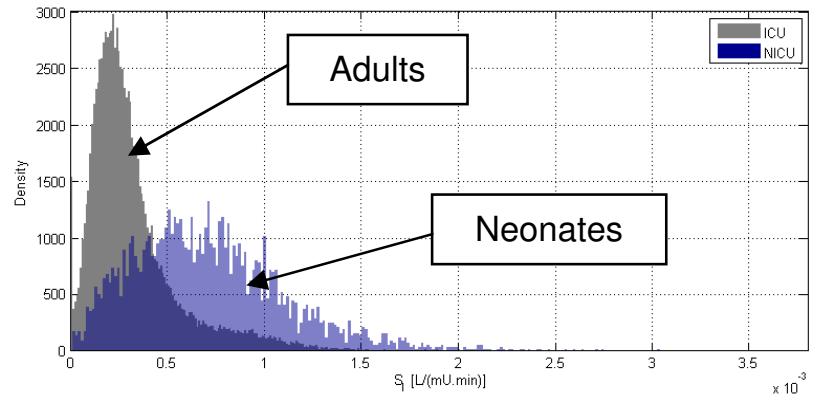

Fig 4. Whole-cohort insulin sensitivity distributions for adults (44,386 hours) and neonates (3,567 hours).

- Whole-cohort constant insulin sensitivity: The median value of insulin sensitivity for the entire cohort is used by the controller for all patients. This method assumes both that all patients exhibit the same level of response to insulin, and that insulin response does not exhibit any temporal variation, ignoring both inter- and intra- patient effects.

Finally, model-based control results in adults are compared to the simulations using the SPRINT protocol - a paper-based protocol designed to mimic model-based control and used clinically since August 2005 (Chase et al., 2008). SPRINT uses 1-2 hourly BG measurement and intervention intervals and modulates both insulin infusion rates and nutritional inputs for control to a 4-6 mmol/L target band.

Retrospective dextrose input profiles and constant two-hourly BG measurement and control interval timing was used for the neonatal cohort simulations, and SPRINT nutrition and BG measurement protocols were used for adult simulations. Bolus-based insulin delivery was simulated for adults and infusion delivery was used for the neonates. These choices reflect typical clinical implementation methods.

\section{RESULTS}

Empirical density functions of fitted insulin sensitivity for
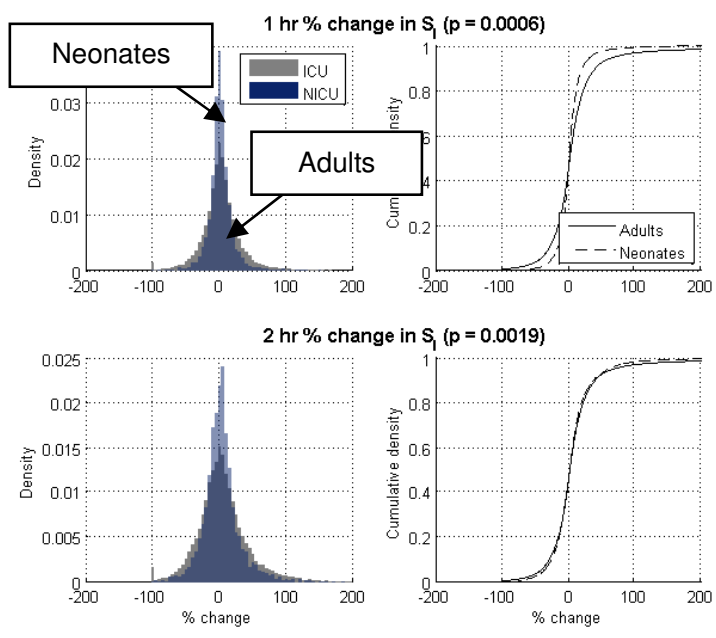

Fig 5. Percentage change in $S_{I}$ over hourly (top) and two-hourly (bottom) intervals for adults and neonates. 
Table 1. Simulated control for adult patients $(\mathrm{N}=393)$ with variations in controller-assumed insulin sensitivity

\begin{tabular}{|c|c|c|c|c|}
\hline & \multicolumn{3}{|c|}{ Model-based control } & \multirow{2}{*}{ SPRINT } \\
\hline & Adaptive control & Per-patient constant & Cohort constant & \\
\hline Total hours: & 48,697 hours & 48,721 hours & 48,759 hours & 48,701 hours \\
\hline Num BG measurements: & 29,202 & 32,641 & 35,036 & 29,376 \\
\hline BG median [IQR] (mmol/L): & $5.5[4.9-6.6]$ & $5.5[4.5-7.3]$ & $5.3[4.1-7.6]$ & $5.9[5.1-6.9]$ \\
\hline BG mean (geometric) (mmol/L): & 5.8 & 5.8 & 5.6 & 6.0 \\
\hline BG SD (geometric) (mmol/L): & 1.3 & 1.4 & 1.5 & 1.3 \\
\hline$\%$ BG within $4.0-6.1 \mathrm{mmol} / \mathrm{L}$ & 60.3 & 46.6 & 38.7 & 51.5 \\
\hline$\%$ BG within $4.0-8.0 \mathrm{mmol} / \mathrm{L}$ & 83.1 & 65.8 & 54.1 & 82.1 \\
\hline$\% \mathrm{BG}<4.0 \mathrm{mmol} / \mathrm{L}$ & 4.8 & 14.0 & 23.2 & 4.8 \\
\hline$\% \mathrm{BG}<2.2 \mathrm{mmol} / \mathrm{L}$ & 0.05 & 0.33 & 1.03 & 0.04 \\
\hline Num patients $<2.2 \mathrm{mmol} / \mathrm{L}$ & 11 & 29 & 67 & 12 \\
\hline Median insulin rate [IQR] (U/hr): & $5.6[2.3-6.0]$ & $4.5[1.9-6.0]$ & $4.5[2.3-6.0]$ & $3.0[2.0-3.0]$ \\
\hline Median glucose rate [IQR] (g/hour): & $5.2[3.9-6.5]$ & $5.2[3.3-6.5]$ & $5.9[2.6-6.5]$ & $5.2[3.3-5.9]$ \\
\hline
\end{tabular}

neonates and adult patients are compared in Fig 4. The median insulin sensitivity for neonates was $0.68 \times 10^{-3}$ $\mathrm{L} /(\mathrm{mU} . \mathrm{min})$, compared to $0.24 \times 10^{-3} \mathrm{~L} /(\mathrm{mU} . \mathrm{min})$ for adults, and these values were used for the respective whole-cohort constant insulin sensitivity control simulation studies. The $5 \%-95 \%$ data interval was $[0.17-1.70] \times 10^{-3} \mathrm{~L} /(\mathrm{mU} . \mathrm{min})$ for neonates and $[0.06-0.79] \times 10^{-3} \mathrm{~L} /(\mathrm{mU} . \mathrm{min})$ for adults.

The distributions of hourly insulin sensitivity variation between adults and neonates are presented in Fig.5. NICU patients show significantly less intra-patient temporal variation in insulin sensitivity. This result, coupled with the wider overall cohort distribution in insulin sensitivity suggests that NICU patients exhibit less intra-patient variation and higher inter-patient variation in response to insulin compared to adults. This result suggests the relative
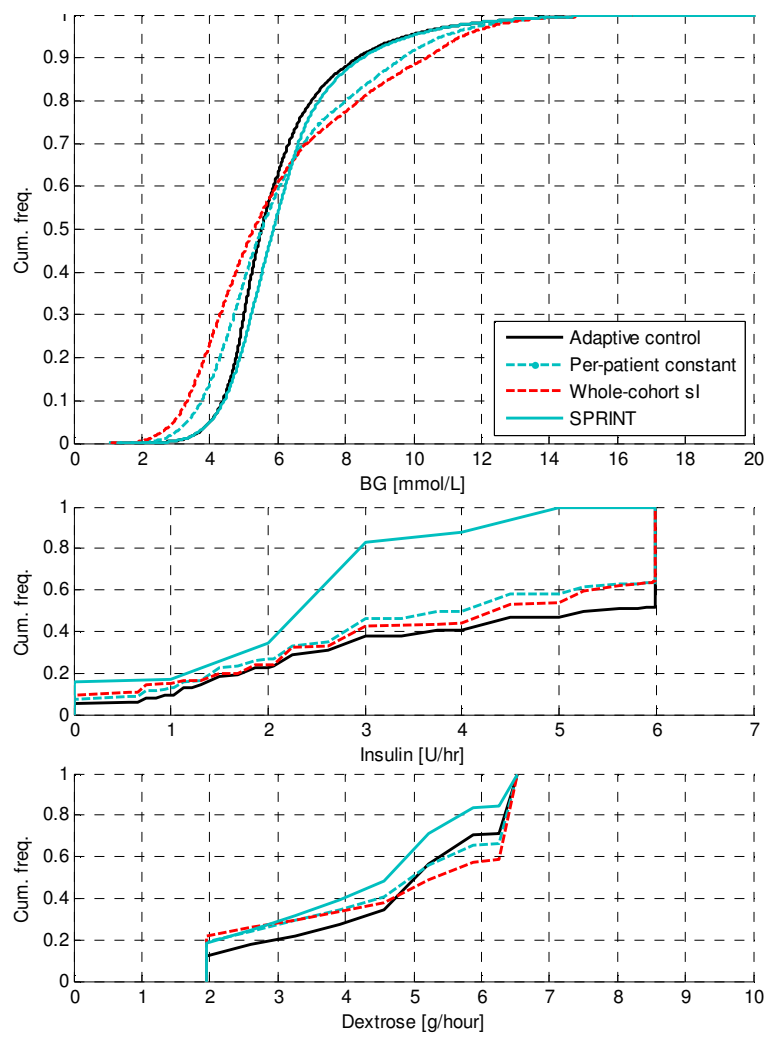

Fig. 6. Simulated distribution of BG (top), insulin usage (middle) and dextrose administration (bottom) for adults. importance of identifying per-patient response to insulin inputs may be different between the two cohorts.

Table 1 presents the glycaemic control performance for adults in simulation of model-based control with assumptions placed on controller-assumed insulin sensitivity. The median BG concentration is similar for all versions of model-based control. However increased variability is evident, as shown by the wider inter-quartile ranges and larger geometric standard deviations. This increased glycaemic variability is shown more dramatically by deteriorating time within target glycaemic ranges and increases in number of measurements below hypoglycaemic threshold and number of patients experiencing hypoglycaemia. This result is also evident in Fig. 6, which shows similar median BG for all model-based controllers, with the tightest spread of BG belonging to the adaptive control method. The per-patient constant and cohortconstant results exhibit flatter distributions, with significant hypoglycaemia. The results for SPRINT in Table 1 show glycaemic control performance with respect to \%BG within the target range is superior to the per-patient constant controller, but less effective than the adaptive controller, where SPRINT achieved $51.5 \%$ within the target band compared to $46.6 \%$ and $60.3 \%$ for per-patient constant and
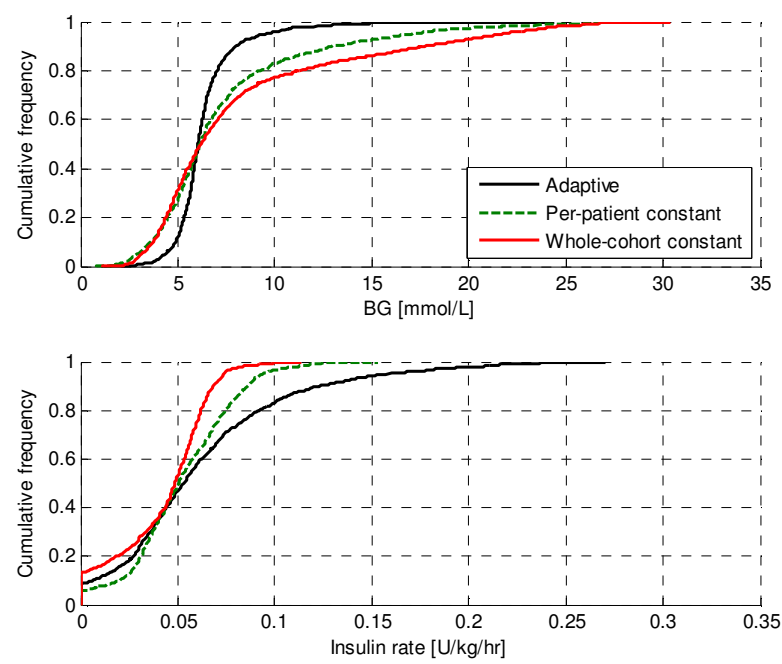

Fig. 7. Simulated distribution of blood glucose concentration and insulin usage for neonatal patients and model-based controllers. 
Table 2. Simulated control for neonatal patients $(\mathrm{N}=25)$ with variations in controller-assumed insulin sensitivity

\begin{tabular}{|c|c|c|c|}
\cline { 2 - 4 } \multicolumn{1}{c|}{} & \multicolumn{3}{c|}{ Model-based control } \\
\cline { 2 - 4 } \multicolumn{1}{c|}{} & Adaptive control & Per-patient constant & 45.6 \\
\%BG within $4.0-7.0 \mathrm{mmol} / \mathrm{L}$ & 76.9 & 50.1 & 54.5 \\
\%BG within $4.0-8.0 \mathrm{mmol} / \mathrm{L}$ & 87.1 & 60.1 & 13.7 \\
\%BG $<4.0 \mathrm{mmol} / \mathrm{L}$ & 2.8 & 13.4 & 1.4 \\
\%BG $<2.6 \mathrm{mmol} / \mathrm{L}$ & 0.3 & 3.0 & $6.2[4.7-9.2]$ \\
BG median $[\mathrm{IQR}](\mathrm{mmol} / \mathrm{L})$ & $6.1[5.5-6.7]$ & $0.2[4.9-8.3]$ & $0.049[0.028-0.060]$ \\
\hline
\end{tabular}

cohort constant control respectively.

Table 2 and Fig. 7 present the results of control simulations on the neonatal cohort. Similar loss of control performance is exhibited for controllers that assume constant insulin sensitivity. Especially evident in Fig. 7 is that the tightest, most vertical BG distribution is associated with adaptive control, yet the opposite pattern is present for insulin rates, where the tightest spread of values is for the whole-cohort constant $S_{I}$ controller. Thus, the constant controllers tend to vary insulin rates less, allowing patient variability to flow through to glycaemic levels, whereas adaptive control stops patient variability flowing through to $B G$ levels by varying insulin rates in accordance to sensitivity to insulin.

\section{DISCUSSION}

Reductions in glycaemic control performance were observed when using controllers that used constant assumptions for insulin sensitivity, and is consistent across both adult and neonatal cohorts. Interestingly, median BG values are similar for all controllers tested, and the reduction in performance was captured in increased glycaemic variability as measured by higher standard deviations, lower times in target bands, higher hypoglycaemia and flatter cumulative distribution curves. This result suggests that designing and analysing glycaemic control with respect to a target median level of glycaemia may miss important information about variability.

The effects of low blood glucose levels are considered much more serious and immediate than high blood sugar levels. Thus, there has been a trend to aim for higher BG levels to avoid hypoglycaemia, whereas a more effective alternative method may be to use protocols that adequately account for variability and provide tighter control, achieving the goals of glucose control to within healthy physiological ranges without increased risk of hypoglycaemia. Importantly, it may be time in a glycaemic control band (eg: $4.4-6.1 \mathrm{mmol} / \mathrm{L}$ ) that determines outcome, rather than a target median, as physiologically it is consistent, controlled glycaemic values that ameliorate the negative effects of hyperglycaemia.

The adaptive controller generally fed an increased amount of glucose compared to non-adaptive controllers, and coupled this with more aggressive use of insulin. The amount of insulin used is a non-linear function of glucose input and glycaemic level, and is influenced by the insulin effect saturation parameter $\left(\alpha_{G}\right)$. This result is also tied to the nutrition heuristics developed for SPRINT, where lower glucose levels are more likely to result in increased glucose inputs, and may be further modified to meet clinical requirements for glucose administration and insulin limits. Insulin effect saturation is not apparent in neonates at physiologic levels (Farrag et al., 1997), thus increases in insulin usage for tighter control are less evident.

SPRINT was designed to be a low-cost, easily implemented paper-based protocol that implemented model-based control. The practicalities of clinical use means that insulin rates are thus discretised to whole-unit amounts, whereas model-based control allows a finer degree of resolution, as well as the ability to capture a broader range of patient dynamics. However, the SPRINT design goal to emulate adaptive model-based control is evidenced by its superior performance to the constant $S_{I}$ versions of model-based control.

The difference in inter- and intra-patient variability between critically ill adults and neonates may in part be explained by infant growth and development. Endogenous glucose control systems are still developing in preterm infants and insulindependent tissues are increasing. Thus, overall resistance to insulin tends to decrease over time.

Insulin sensitivity $\left(S_{I}\right)$ has been chosen in this study to quantify response to insulin as it is identifiable from clinically available measurements. Thus, it represents several physiological processes, such as suppression of endogenous glucose production and increase in tissue glucose uptake that cannot be identified separately. However, the overall conclusion of the requirement of accounting for patient response over time as a major contributor to tight glycaemic control stands as a result independent of the specific model chosen. More advanced models, if able to be identified in clinical-real time, can provide advantages by improving predictive performance, and thus control quality.

Model-based control derives interventions from an estimate of patient metabolic state. This estimate may be influenced by errors, such as noise in BG sensors, missing patient data and user error, increasing the observed variability in patient response. Control methods need to provide a balance between aggressiveness in meeting glycaemic targets and robustness to noise and errors to maintain patient safety against hypoglycaemia.

Identifying the contributing factors to variation in glycaemia response may be useful in optimising several aspects of therapy in critical care and beyond. Optimisations may focus on the cost of measurement and control interventions in terms of pump expense for ambulatory individuals, multiple daily injections, time required to devote to maintaining blood sugar levels each day for diabetic patients. Also the need to conserve blood volumes in neonates, as well as minimise 
handling to prevent excessive infant stress emphasises the importance of optimising the balance between clinical effort and safe, robust control.

\section{CONCLUSIONS}

Tight glycaemic control requires adequately addressing interand intra- patient variability. Tracking patient metabolic response over time and titrating interventions to estimates of insulin sensitivity provides significant improvements in control performance over fixed protocols for both adults and neonates. This result clearly defines the differences seen in several published trials in outcome glycaemia and variability due to these variations in patient behaviour. Hence, it highlights the need to better account for these variations to maximise the opportunity for successful TGC.

\section{REFERENCES}

Alaedeen, D. I., Walsh, M. C. \& Chwals, W. J. 2006. Total parenteral nutrition-associated hyperglycemia correlates with prolonged mechanical ventilation and hospital stay in septic infants. J Pediatr Surg, 41, 239-44; discussion 239-44.

Beardsall, K., Vanhaesebrouck, S., Ogilvy-Stuart, A. L., Ahluwalia, J. S., Vanhole, C., Palmer, C., Midgley, P., Thompson, M., Cornette, L., Weissenbruch, M., Thio, M., De Zegher, F. \& Dunger, D. 2007. A randomised controlled trial of early insulin therapy in very low birth weight infants, "NIRTURE" (neonatal insulin replacement therapy in Europe). BMC pediatrics, 7, 29.

Bistrian, B. R. 2001. Hyperglycemia and infection: which is the chicken and which is the egg? JPEN J Parenter Enteral Nutr, 25, 180-181.

Capes, S. E., Hunt, D., Malmberg, K. \& Gerstein, H. C. 2000. Stress hyperglycaemia and increased risk of death after myocardial infarction in patients with and without diabetes: a systematic overview. Lancet, 355, 773-778.

Chase, J., Shaw, G. M., Wong, X. W., Lotz, T., Lin, J. \& Hann, C. E. 2006. Model-based glycaemic control in critical care - a review of the state of the possible. Biomedical Signal Processing and Control, 1, 3-21.

Chase, J. G., Hann, C. E., Shaw, G. M., Wong, X. W., Lin, J., Lotz, T., Le Compte, A. J. \& Lonergan, T. 2007. An overview of glycemic control in critical care - relating performance and clinical results. Journal of Diabetes Science and Technology, 1, 82-91.

Chase, J. G., Shaw, G., Le Compte, A., Lonergan, T., Willacy, M., Wong, X.-W., Lin, J., Lotz, T., Lee, D. \& Hann, C. 2008. Implementation and evaluation of the SPRINT protocol for tight glycaemic control in critically ill patients: a clinical practice change. Critical Care, 12, R49.

Cowett, R. M., Oh, W. \& Schwartz, R. 1983. Persistent glucose production during glucose infusion in the neonate. J Clin Invest, 71, 467-75.

Egi, M., Bellomo, R., Stachowski, E., French, C. J. \& Hart, G. 2006. Variability of blood glucose concentration and short-term mortality in critically ill patients. Anesthesiology, 105, 244-52.
Ertl, T., Gyarmati, J., Gaal, V. \& Szabo, I. 2006. Relationship between hyperglycemia and retinopathy of prematurity in very low birth weight infants. Biology of the neonate, $89,56-9$.

Farrag, H. M., Nawrath, L. M., Healey, J. E., Dorcus, E. J., Rapoza, R. E., Oh, W. \& Cowett, R. M. 1997. Persistent glucose production and greater peripheral sensitivity to insulin in the neonate vs. the adult. Am J Physiol, 272, E86-93.

Hann, C. E., Chase, J. G., Lin, J., Lotz, T., Doran, C. V. \& Shaw, G. M. 2005. Integral-based parameter identification for long-term dynamic verification of a glucose-insulin system model. Comput Methods Programs Biomed, 77, 259-270.

Hays, S. P., Smith, B. \& Sunehag, A. L. 2006. Hyperglycemia Is a Risk Factor for Early Death and Morbidity in Extremely Low Birth-Weight Infants. Pediatrics, 118, 1811-1818.

Le Compte, A., Chase, J., Lynn, A., Hann, C., Shaw, G., Wong, X. \& Lin, J. 2009. Blood Glucose Controller for Neonatal Intensive Care: Virtual trials development and 1 st clinical trials. Journal of Diabetes Science and Technology, 3, 1066-1081.

Lonergan, T., Lecompte, A., Willacy, M., Chase, J. G., Shaw, G. M., Wong, X. W., Lotz, T., Lin, J. \& Hann, C. E. 2006. A simple insulin-nutrition protocol for tight glycemic control in critical illness: development and protocol comparison. Diabetes Technol Ther, 8, 191-206.

Mccowen, K. C., Malhotra, A. \& Bistrian, B. R. 2001. Stressinduced hyperglycemia. Crit Care Clin, 17, 107-124.

Mitanchez-Mokhtari, D., Lahlou, N., Kieffer, F., Magny, J.F., Roger, M. \& Voyer, M. 2004. Both Relative Insulin Resistance and Defective Islet \{beta\}-Cell Processing of Proinsulin Are Responsible for Transient Hyperglycemia in Extremely Preterm Infants. Pediatrics, 113, 537-541.

Preiser, J. C. \& Devos, P. 2007. Clinical experience with tight glucose control by intensive insulin therapy. Crit Care Med, 35, S503-7.

Raney, M., Donze, A. \& Smith, J. R. 2008. Insulin infusion for the treatment of hyperglycemia in low birth weight infants: examining the evidence. Neonatal Netw, 27, 127-40.

Schultz, M. J., De Graaff, M. J., Kuiper, M. A. \& Spronk, P. E. 2008. The new Surviving Sepsis Campaign recommendations on glucose control should be reconsidered. Intensive Care Med, 34, 779-80; author reply 781-2.

Van Den Berghe, G., Wouters, P., Weekers, F., Verwaest, C., Bruyninckx, F., Schetz, M., Vlasselaers, D., Ferdinande, P., Lauwers, P. \& Bouillon, R. 2001. Intensive insulin therapy in the critically ill patients. N Engl J Med, 345, 1359-1367.

Wong, X. W., Chase, J. G., Shaw, G. M., Hann, C. E., Lotz, T., Lin, J., Singh-Levett, I., Hollingsworth, L. J., Wong, O. S. \& Andreassen, S. 2006. Model predictive glycaemic regulation in critical illness using insulin and nutrition input: a pilot study. Med Eng Phys, 28, 665-81. 